\title{
Heisenberg Form of Uncertainty Relations
}

\author{
Mohammed Yousif ${ }^{1}$, Mohammed Ali Basheir ${ }^{2}$, Emadaldeen Abdalrahim $^{3}$ \\ ${ }^{1}$ Sudan University of Science and Technology (SUST), Math Department, College of Science, Khartoum, Sudan \\ ${ }^{2}$ Math Department, College of Science, ALNeelein University, Khartoum, Sudan \\ ${ }^{3}$ Sudan University of Science and Technology (SUST), Math Department, College of Science, Khartoum, Sudan
}

Email address:

mohammed.yousifphd@gmail.com (M. Yousif), Mabashir@hotmail.com (M. A. Basheir), emadsust@gmail.com (E. Abdalrahim)

\section{To cite this article:}

Mohammed Yousif, Mohammed Ali Basheir, Emadaldeen Abdalrahim. Heisenberg Form of Uncertainty Relations. American Journal of Applied Mathematics. Vol. 3, No. 6, 2015, pp. 321-326. doi: 10.11648/j.ajam.20150306.22

\begin{abstract}
This paper, deals with the uncertainty relation for photons. In [Phys.Rev.Let.108, 140401 (2012)], and [1] the uncertainty relation was obtained as a sharp inequality by using the energy distribution on space. The relation we obtain here is an alternative to the one given in [Phys.Rev.Let.108, 140401 (2012)] by the use of the position of the center of the energy operator. The fact that the components of the center is non commutative affected the right hand side of the Heisenberg inequality. But this resolved by the increase of the photon energy. Furthermore we study the uncertainty of Heisenberg with respect to angular momentum and Foureir. We end the paper by giving some examples.
\end{abstract}

Keywords: Uncertainty Relation for Photons, Quantum Mechanics of Photons, Foureir Theory

\section{Introduction}

As in the standard Heisenberg form of uncertainty relation for photons is somewhat difficult identity its position operator. However, it is apparently, photon can be influenced by the spread of momentum and the extension in space that represent the famous Heisenberg phrase "Je genauer der Ort bestimmt ist, desto ungenauer ist der Impuls bekannt und umgekehrt."

The photon uncertainty relations is mainly divided into two defined notions: the photon wave function as mentioned in momentum space and then the energy density of the quantized electromagnetic field. Thus, when the second momentum is applied, it is generated the following form[1]

$$
\Delta r \Delta p \geq 4 h
$$

This work is aimed to construct a definition for the uncertainty of the photons position so that can be analogous to the standard definition, which done by Iwo BialynickiBirula, Zofia Bialynicka-Birula Heisenberg uncertainty relations for photons (2012) [1]. This to illustrate the importance of $\widehat{\mathrm{R}}$ as the center of energy to the first momentum. Therefore, this method is going to serve in making uncertainty relation as close as the original form of Heisenberg,

$$
\sqrt{\Delta R^{2}} \sqrt{\Delta P^{2}}>\frac{d}{2} h
$$

Where $\mathrm{d}$ is the number of dimensions. A characteristic feature of the uncertainty relation for photons is that the lefthand side in this inequality in two and in three dimensions is never equal to $\mathrm{dh} / 2$, but it tends to this limit with the increase of the average photon momentum. Only in the infinite-momentum frame is the uncertainty relation for photons the same as for nonrelativistic massive particles. However, in one dimension, the inequality (2) is saturated so that in this case there is no difference between photons and massive nonrelativistic particles.

We also prove the following sharp inequality

$$
\sqrt{\langle\hat{R} . \hat{R}\rangle} \sqrt{\langle\hat{P} . \hat{P}\rangle} \geq \frac{3}{2} h \sqrt{1+\frac{4 \sqrt{5}}{9}}
$$

In nonrelativistic quantum mechanics, the inequalities obeyed by the two measures of uncertainty, $\Delta \mathrm{R}^{2} \Delta \mathrm{P}^{2}$ and $\langle\widehat{R} . \widehat{R}\rangle\langle\widehat{P} . \widehat{P}\rangle$, are completely equivalent. They have equal lower bounds and they are both saturated by Gaussian functions. This equivalence does not hold for photons. Nevertheless, the two inequalities are intimately related. We shall first prove (3) and then use the information about the photon states that saturate this inequality to elucidate the intricate properties of the inequality (2).

Study has been done by Schwinger, he came up with the rough estimate that the lower bound of $\Delta \mathrm{R}^{2} \Delta \mathrm{P}^{2}$ is of the order of $h^{2}$.

This work is endeavored to derive a related uncertainty 
relation for photons beams. Making use of Coherent states of the electromagnetic field to find a description of such beams in the limit of a large number of photons so as to prove the sharp inequality as follows[1], [3]

$$
\sqrt{\Delta \mathrm{R}^{2}} \sqrt{\Delta \mathrm{P}^{2}} \geq \frac{3}{2} \mathrm{~h} \sqrt{1+\frac{4 \sqrt{2}}{9}}
$$

And we find the mode functions of the coherent states that saturate this inequality.

The nonexistence of the local photon density in configuration space is due to the fact that in quantum electrodynamics the operator of the total number of photons $\widehat{\mathrm{N}}$ involves not a single but a double integral:

$$
\begin{gathered}
\widehat{N}=\frac{1}{4 \pi^{2} h c} \int d^{3} r \int d^{3} r^{\prime} \times:\left[\frac{\widehat{D}(r, t) \cdot \widehat{D}\left(r^{\prime}, t\right)}{\varepsilon\left|r-r^{\prime}\right|^{2}}+\frac{\widehat{B}(r, t) \cdot \hat{B}\left(r^{\prime}, t\right)}{\mu\left|r-r^{\prime}\right|^{2}}\right] \\
=\frac{1}{2 \pi^{2} h c} \int d^{3} r \int d^{3} r^{\prime}:\left[\frac{\hat{F}^{\dagger}(r, t) \cdot \hat{F}\left(r^{\prime}, t\right)}{\left|r-r^{\prime}\right|^{2}}\right]
\end{gathered}
$$

We use systematically the Riemann-Silberstein vector (the RS vector)

$$
\hat{F}(r, t)=\frac{\widehat{D}(r, t)}{\sqrt{2 \epsilon}}+i \frac{\hat{B}(r, t)}{\sqrt{2 \mu}}
$$

Which will allow us to write many formulas in a compact form. The normal ordering removes the (infinite) contribution from the vacuum state[1]. In contrast to the total-number operator, the total-energy operator of the electromagnetic field $\widehat{H}$ (the Hamiltonian) is an integral of a local density

$$
\widehat{H}=\int d^{3} r \hat{\varepsilon}(r, t)
$$

Where

$$
\hat{\varepsilon}(r, t)=: \hat{F}^{\dagger}(r, t) \cdot \hat{F}\left(r^{\prime}, t\right):
$$

The center of the energy operator can be introduced in any relativistic theory. All we need for this construction is the set of generators of the Poincar'e group. The Poincar'e group is the group of Minkowski spacetime isometries. It is a ten dimensional noncompact Lie group. The abelian group of translations is a normal subgroup, while the Lorentz group is also a subgroup, the stabilizer of the origin. The Poincar'e group itself is the minimal subgroup of the affine group which includes all translations and Lorentz translations. More precisely, it is a semi direct product of the translations and Lorentz group.

We define the operator $\hat{R}$ as follows[1], [15]:

$$
\widehat{R}=\frac{1}{2 \widehat{H}} \widehat{N}+\widehat{N} \frac{1}{2 \widehat{H}}=\frac{1}{\sqrt{\widehat{H}}} \widehat{N} \frac{1}{\sqrt{\widehat{H}}}
$$

Where $\widehat{\mathrm{N}}$ is the first moment of the energy distribution,

$$
\widehat{N}=\int d^{3} r r \hat{\varepsilon}(r, t)
$$

Example(1):

Prove that

$$
\widehat{R}=\frac{1}{2 \widehat{H}} \widehat{N}+\widehat{N} \frac{1}{2 \widehat{H}}=\frac{1}{\sqrt{\widehat{H}}} \widehat{N} \frac{1}{\sqrt{\widehat{H}}} .
$$

Proof.

The symmetrization in (9) is necessary to obtain a Hermitian operator. The inverse of the Hamiltonian is well defined, provided we exclude the vacuum state. The spectrum of the Hamiltonian is nonnegative, therefore the positive square root is unique. The significance of $\widehat{N}$ is further underscored by its being the generator of Lorentz transformations. Since the operators $\widehat{H}$ and $\widehat{N}$ do not commute (the energy changes under Lorentz transformations), the equivalence of the two forms of $\hat{R}$ in (9) is not obvious and to prove the equality of the two forms of $\hat{R}$ in (9) we will first prove the following lemma

$$
\text { if }[\widehat{H}, \hat{C}]=0 \text { then }[\sqrt{\widehat{H}}, \hat{C}]=0
$$

In the proof, we use the fact that the eigenvectors of the Hamiltonian form a basis. Acting on an arbitrary state in this basis $|E\rangle$ (excluding the vacuum), we have:

$$
(\sqrt{\widehat{H}}, \sqrt{E})=[\sqrt{\widehat{H}}, \hat{C}]|E\rangle=[\widehat{H}, \hat{C}]|E\rangle=0
$$

Since the factor $(\sqrt{\widehat{\mathrm{H}}}, \sqrt{\mathrm{E}})$ does not vanish, it can be dropped and the validity of the lemma is established [1].

Next, we use the commutation relations between the Hamiltonian and the generator of the Lorentz transformations

$$
[\widehat{H}, \widehat{N}]=-i \hbar \widehat{P}
$$

To obtain:

$$
\left[\widehat{H},\left[\frac{1}{\sqrt{\widehat{H}}} \widehat{N} \frac{1}{\sqrt{\widehat{H}}}, \frac{1}{\sqrt{\widehat{H}}}\right]\right]=\left[\frac{1}{\sqrt{\widehat{H}}}[\widehat{H}, \widehat{N}] \frac{1}{\sqrt{\overparen{H}}}, \frac{1}{\sqrt{\widehat{H}}}\right]=\frac{\hbar}{i}\left[\frac{\hat{P}}{\widehat{H}}, \frac{1}{\sqrt{\widehat{H}}}\right]=0(14)
$$

Finally, using the lemma, we may replace $\widehat{H}$ by $\sqrt{\widehat{H}}$ in the first term and expand the resulting double commutator:

$$
0=\left[\sqrt{\widehat{\mathrm{H}}}\left[\frac{1}{\sqrt{\widehat{H}}} \widehat{N} \frac{1}{\sqrt{\overparen{H}}}, \frac{1}{\sqrt{\widehat{H}}}\right]\right]=\frac{1}{\widehat{H}} \widehat{N}+\widehat{N} \frac{1}{\widehat{H}}-2 \frac{1}{\sqrt{\widehat{H}}} \widehat{N} \frac{1}{\sqrt{\widehat{H}}}
$$

The vanishing of the difference of two expressions for $\hat{R}$ appearing in (9) means that they are equal.

\section{The Generalized Uncertainty Relation}

Earlier we learned about the famous Hiesenberg uncertainty principle which relates the uncertainly in position to that of momentum via from formula (1):

$$
\Delta \mathrm{x} \Delta \mathrm{p} \geq \frac{\hbar}{2}
$$

We now generalize this relation to any two arbitrary operators A and B. First, we recall that in a given state $|\psi\rangle$, the mean or expectation value of an operator $o$ is found to be[6]:

$$
\langle 0\rangle=\langle\psi|0| \psi\rangle
$$


Now let's consider the standard deviation or uncertainty for two operators A and B:

$$
\begin{aligned}
& (A)^{2}=\left\langle(A-\langle A\rangle)^{2}\right\rangle \\
& (B)^{2}=\left\langle(B-\langle B\rangle)^{2}\right\rangle
\end{aligned}
$$

Using $\langle 0\rangle=\langle\psi|0| \psi\rangle$ we can rewrite these two equations as:

$$
\begin{aligned}
& (\Delta \mathrm{A})^{2}=\left\langle(\mathrm{A}-\langle\mathrm{A}\rangle)^{2}\right\rangle=\left\langle\psi\left|\langle\mathrm{A}-\langle\mathrm{A}\rangle\rangle^{2}\right| \psi\right\rangle \\
& (\Delta B)^{2}=\left\langle(B-\langle B\rangle)^{2}\right\rangle=\left\langle\psi\left|\langle B-\langle B\rangle\rangle^{2}\right| \psi\right\rangle
\end{aligned}
$$

We now define the following kets:

$$
\begin{aligned}
& |\mathrm{X}\rangle=(\mathrm{A}-\langle\mathrm{A}\rangle)|\psi\rangle \\
& |\Phi\rangle=(\mathrm{B}-\langle\mathrm{B}\rangle)|\psi\rangle
\end{aligned}
$$

This allows us to write:

$$
\begin{aligned}
& (\Delta \mathrm{A})^{2}=\left\langle(\mathrm{A}-\langle\mathrm{A}\rangle)^{2}\right\rangle=\left\langle\psi\left|\langle\mathrm{A}-\langle\mathrm{A}\rangle\rangle^{2}\right| \psi\right\rangle=\langle\mathrm{X} \mid \mathrm{X}\rangle \\
& (\Delta \mathrm{B})^{2}=\left\langle(\mathrm{B}-\langle\mathrm{B}\rangle)^{2}\right\rangle=\left\langle\psi\left|\langle\mathrm{B}-\langle\mathrm{B}\rangle\rangle^{2}\right| \psi\right\rangle=\langle\Phi \mid \Phi\rangle
\end{aligned}
$$

Now consider the product of these terms[6], [11]:

$$
(\Delta \mathrm{A})^{2}(\Delta \mathrm{B})^{2}=\langle\mathrm{X} \mid \mathrm{X}\rangle\langle\Phi \mid \Phi\rangle
$$

The Schwartz inequality tells us that:

$$
\langle\mathrm{X} \mid \mathrm{X}\rangle\langle\Phi \mid \Phi\rangle \geq|\langle\mathrm{X} \mid \Phi\rangle|^{2}=\langle\mathrm{X} \mid \Phi\rangle\langle\Phi \mid \mathrm{X}\rangle
$$

Remember that the inner product formed by a ket and a bra is just a complex number, so $|\langle\mathrm{X} \mid \Phi\rangle|^{2}=|\mathrm{Z}|^{2}=\mathrm{ZZ}^{*}$ For any complex number $\mathrm{z}$, we have:

$$
\mathrm{ZZ}^{*}=\operatorname{Re}(\mathrm{z})^{2}+\operatorname{Im}(\mathrm{z})^{2} \geq \operatorname{Im}(\mathrm{z})^{2}=\left(\frac{\mathrm{z}+\overline{\mathrm{z}}}{2 \mathrm{i}}\right)^{2}
$$

In this case we have:

$$
\begin{gathered}
\langle\mathrm{X} \mid \Phi\rangle=\langle\psi|(\mathrm{A}-\langle\mathrm{A}\rangle)(\mathrm{B}-\langle\mathrm{B}\rangle)| \psi\rangle \\
=\langle\psi|\mathrm{AB}-\mathrm{A}\langle\mathrm{B}\rangle-\langle\mathrm{A}\rangle \mathrm{B}+\langle\mathrm{A}\rangle\langle\mathrm{B}\rangle| \psi\rangle \\
=\langle\psi|\mathrm{AB}| \psi\rangle-\langle\psi|\mathrm{A}\langle\mathrm{B}\rangle| \psi\rangle-\langle\psi|\langle\mathrm{A}\rangle \mathrm{B}| \psi\rangle+\langle\psi|\langle\mathrm{A}\rangle\langle\mathrm{B}\rangle| \psi\rangle
\end{gathered}
$$

Now $\langle A\rangle$, the expectation value of an operator, is just a number. So we can pull it out of each term giving:

$$
\begin{gathered}
\langle\psi|\mathrm{AB}| \psi\rangle-\langle\psi|\mathrm{A}| \psi\rangle\langle\mathrm{B}\rangle-\langle\mathrm{A}\rangle\langle\psi|\mathrm{B}| \psi\rangle+\langle\psi|\langle\mathrm{A}\rangle\langle\mathrm{B}\rangle| \psi\rangle \\
=\langle\psi|\mathrm{AB}| \psi\rangle-\langle\mathrm{A}\rangle\langle\mathrm{B}\rangle-\langle\mathrm{A}\rangle\langle\mathrm{B}\rangle+\langle\psi|\langle\mathrm{A}\rangle\langle\mathrm{B}\rangle| \psi\rangle \\
=\langle\mathrm{AB}\rangle-2\langle\mathrm{~A}\rangle\langle\mathrm{B}\rangle+\langle\psi|\langle\mathrm{A}\rangle\langle\mathrm{B}\rangle| \psi\rangle
\end{gathered}
$$

Now the expectation value of the mean, which is again just a number, is simply the mean back again, i.e.

$$
\langle\psi|\langle\mathrm{A}\rangle\langle\mathrm{B}\rangle| \psi\rangle=\langle\langle\mathrm{A}\rangle\langle\mathrm{B}\rangle\rangle=\langle\mathrm{A}\rangle\langle\mathrm{B}\rangle
$$

So, finally we have:

$$
\begin{aligned}
& \langle\mathrm{X} \mid \Phi\rangle=\langle\psi|(\mathrm{A}-\langle\mathrm{A}\rangle)(\mathrm{B}-\langle\mathrm{B}\rangle)| \psi\rangle \\
& =\langle\mathrm{AB}\rangle-2\langle\mathrm{~A}\rangle\langle\mathrm{B}\rangle+\langle\psi|\langle\mathrm{A}\rangle\langle\mathrm{B}\rangle| \psi\rangle
\end{aligned}
$$

$$
=\langle A B\rangle-2\langle A\rangle\langle B\rangle+\langle A\rangle\langle B\rangle=\langle A B\rangle-\langle A\rangle\langle B\rangle
$$

Following a similar procedure, we can show that:

$$
\langle\Phi \mid \mathrm{X}\rangle=\langle\psi|(\mathrm{B}-\langle\mathrm{B}\rangle)(\mathrm{A}-\langle\mathrm{A}\rangle)| \psi\rangle=\langle\mathrm{BA}\rangle-\langle\mathrm{A}\rangle\langle\mathrm{B}\rangle
$$

Putting everything together allows us to find an uncertainty relation for A and B. First we have:

$$
(\Delta \mathrm{A})^{2}(\Delta \mathrm{B})^{2}=\langle\mathrm{X} \mid \mathrm{X}\rangle\langle\Phi \mid \Phi\rangle \geq|\langle\mathrm{X} \mid \Phi\rangle|^{2}=\langle\mathrm{X} \mid \Phi\rangle\langle\Phi \mid \mathrm{X}\rangle
$$

Recalling that[6],

$$
\mathrm{ZZ}^{*}=\operatorname{Re}(\mathrm{z})^{2}+\operatorname{Im}(\mathrm{z})^{2} \geq \operatorname{Im}(\mathrm{z})^{2}=\left(\frac{\mathrm{z}-\overline{\mathrm{z}}}{2 \mathrm{i}}\right)^{2},
$$

We set $\mathrm{Z}^{*}=\langle\Phi \mid \mathrm{X}\rangle$.Then

$$
\begin{gathered}
(\Delta \mathrm{A})^{2}(\Delta \mathrm{B})^{2} \geq|\langle\mathrm{X} \mid \Phi\rangle|^{2}=\left(\frac{\langle\mathrm{X} \mid \Phi\rangle-\langle\Phi \mid \mathrm{X}\rangle}{2 \mathrm{i}}\right) \\
=\left(\frac{(\langle\mathrm{AB}\rangle-\langle\mathrm{A}\rangle\langle\mathrm{B}\rangle)-(\langle\mathrm{BA}\rangle-\langle\mathrm{A}\rangle\langle\mathrm{B}\rangle)}{2 \mathrm{i}}\right)^{2} \\
=\left(\frac{(\langle\mathrm{AB}\rangle-\langle\mathrm{A}\rangle\langle\mathrm{B}\rangle)-\langle\mathrm{BA}\rangle+\langle\mathrm{A}\rangle\langle\mathrm{B}\rangle}{2 \mathrm{i}}\right)^{2} \\
=\left(\frac{\langle\mathrm{AB}\rangle-\langle\mathrm{BA}\rangle}{2 \mathrm{i}}\right)^{2} \\
=\left(\frac{\langle\mathrm{AB}-\mathrm{BA}\rangle}{2 \mathrm{i}}\right)^{2} \\
=\left(\frac{\langle[\mathrm{A}, \mathrm{B}]\rangle}{2 \mathrm{i}}\right)^{2}
\end{gathered}
$$

Taking the square root of both sides gives us the generalized uncertainty relation, which applies to any two operators A and B.

Definition: The Uncertainty Relation Given any two operators $\mathrm{A}$ and $\mathrm{B}$ :

$$
\Delta \mathrm{A} \Delta \mathrm{B} \geq \frac{\langle[\mathrm{A}, \mathrm{B}]\rangle}{2 \mathrm{i}}
$$

Where $[\mathrm{A}, \mathrm{B}]$ is the commutator of the operators $\mathrm{A}$ and $\mathrm{B}$. For the operators $\mathrm{X}$ and $\mathrm{P}$, we find that $[\mathrm{X}, \mathrm{P}]=\mathrm{i} \hbar$. So

$$
\frac{[\mathrm{X}, \mathrm{P}]}{2 \mathrm{i}}=\frac{\mathrm{i} \hbar}{2 \mathrm{i}}=\frac{\hbar}{2}
$$

Therefore we obtain the famous Hiesenberg uncertainty principle:

$$
\Delta \mathrm{X} \Delta \mathrm{P} \geq \frac{\hbar}{2}
$$

Also, we can despite all of the differences between the nonrelativistic and relativistic dynamics we may derive a sharp Heisenberg uncertainty relation along one direction, say $\mathrm{x}$, for any relativistic system. This one-dimensional uncertainty relation is based solely on the commutation relations between $\widehat{\mathrm{X}}=\widehat{\mathrm{R}}_{\mathrm{X}}$ and $\widehat{\mathrm{P}}=\widehat{\mathrm{P}}_{\mathrm{X}}$ and has the standard form[1] 


$$
\sqrt{\Delta \mathrm{X}^{2}} \sqrt{\Delta \mathrm{P}^{2}} \geq \frac{1}{2} \mathrm{~h}
$$

where

$$
\begin{aligned}
& \Delta \mathrm{X}^{2}=\left\langle(\Delta \widehat{\mathrm{P}})^{2}\right\rangle, \Delta \widehat{\mathrm{X}}=\widehat{\mathrm{X}}-\langle\widehat{\mathrm{X}}\rangle \\
& \Delta \mathrm{P}^{2}=\left\langle(\Delta \widehat{\mathrm{P}})^{2}\right\rangle, \Delta \widehat{\mathrm{P}}=\widehat{\mathrm{P}}-\langle\widehat{\mathrm{P}}\rangle
\end{aligned}
$$

The one-dimensional uncertainty relation holds for any relativistic quantum system. A simple proof of (39) uses the commutation relations $\left[\widehat{\mathrm{R}}_{\mathrm{i}}, \widehat{\mathrm{P}}_{\mathrm{j}}\right]=\mathrm{ih} \delta_{\mathrm{ij}}$ and the non-negative expectation value of the operator:

$$
\langle(\Delta \widehat{\mathrm{X}}-\mathrm{i} \lambda \Delta \widehat{\mathrm{P}})(\Delta \widehat{\mathrm{X}}+\mathrm{i} \lambda \Delta \widehat{\mathrm{P}})\rangle \geq 0
$$

Where $\lambda$ is an arbitrary real number. The condition that this expression treated as a function of $\lambda$ can have at most one real root gives (39). This inequality is saturated by the quantum state whose state vector satisfies the condition

$$
(\Delta \widehat{\mathrm{X}}-\mathrm{i} \lambda \Delta \widehat{\mathrm{P}})|\Psi\rangle=0 .
$$

The specific form of $|\Psi\rangle$ depends, of course, on the system under study. Note that we may remove the average values $\hbar$ $\langle\widehat{X}\rangle$ and $\hbar\langle\widehat{P}\rangle$ from (43) by choosing $|\Psi\rangle$ in the form[1]

$$
|\Psi\rangle=\exp (\mathrm{i}\langle\widehat{\mathrm{P}}\rangle \widehat{\mathrm{X}} / \mathrm{h}-\mathrm{i}\langle\widehat{\mathrm{X}}\rangle \widehat{\mathrm{P}} / \mathrm{h})\left|\Psi^{\prime}\right\rangle
$$

Since the inequality must hold for all vectors, replacing $|\Psi\rangle$ by $\left|\Psi^{\prime}\right\rangle$ makes no difference and the two forms of the uncertainty relation in one dimension, namely,

$$
\sqrt{\Delta \mathrm{X}^{2}} \sqrt{\Delta \mathrm{P}^{2}} \geq \frac{1}{2} \mathrm{~h} \text { and } \sqrt{\left(\widehat{\mathrm{X}}^{2}\right)} \sqrt{\left(\widehat{\mathrm{P}}^{2}\right)} \geq \frac{1}{2} \mathrm{~h}
$$

are completely equivalent. In nonrelativistic quantum mechanics the equivalence holds in any number of dimensions. A spherically symmetric Gaussian function shifted in the coordinate space by $\langle r\rangle$ and in the momentum space by $\langle p\rangle$ by the unitary transformation of the form (44) will automatically saturate the inequality (2). This equivalence, however, is no longer valid for relativistic systems in three dimensions[1], [10], [7].

To extend our analysis to two and three dimensions, we introduce the dispersion in position that involves two or three components of the center-of-energy vector $\widehat{R}$,

$$
\Delta \mathrm{R}^{2}=\langle\Delta \widehat{\mathrm{R}} \cdot \Delta \widehat{\mathrm{R}}\rangle
$$

Where $\Delta \widehat{\mathrm{R}}=\widehat{\mathrm{R}}-\langle\mathrm{R}\rangle$ and the dispersion in momentum,

$$
\Delta \mathrm{P}^{2}=\langle\Delta \widehat{\mathrm{P}} \cdot \Delta \widehat{\mathrm{P}}\rangle
$$

Where $\Delta \widehat{\mathrm{P}}=\widehat{\mathrm{P}}-\langle\widehat{\mathrm{P}}\rangle$. Following the same procedure as the one used in deriving (39), we obtain (2). The proof is based this time on the expectation value of the following positive operator:

$$
\langle(\Delta \widehat{\mathrm{R}}-\mathrm{i} \lambda \Delta \widehat{\mathrm{P}})(\Delta \widehat{\mathrm{R}}+\mathrm{i} \lambda \Delta \widehat{\mathrm{P}})\rangle>0
$$

In contrast to the one-dimensional case, the inequalities (2) and (48) are not sharp because there is no state vector that is annihilated by all three components of the vector operato $\widehat{A}=$ $\Delta \widehat{\mathrm{R}}+\mathrm{i} \lambda \Delta \widehat{\mathrm{P}}$ and even by two components. This is due to the fact that the commutators $\left[\widehat{\mathrm{R}}_{\mathrm{i}}, \widehat{\mathrm{R}}_{\mathrm{j}}\right]=-\mathrm{ihc} \mathrm{c}^{2} \widehat{\mathrm{H}}^{-1} \widehat{\mathrm{S}}_{\mathrm{ij}} \widehat{\mathrm{H}}^{-1}$ of the components of $\widehat{\mathrm{R}}$ do not vanish. Should there exist a state vector annihilated by $\widehat{A}$, then this vector would also be annihilated by the commutators of the components of $\widehat{A}$. These commutators are proportional to the components of spin. Therefore, for any relativistic quantum system endowed with spin the inequality (2) cannot be saturated.

\section{The Uncertainty Relations for Angular Momentum}

Recalling the generalized uncertainty relation for two operators A and B,

$$
\Delta \mathrm{A} \Delta \mathrm{B} \geq\left|\frac{\left\langle\left[\mathrm{L}_{\mathrm{x}}, \mathrm{L}_{\mathrm{y}}\right]\right\rangle}{2 \mathrm{i}}\right|
$$

we can write down uncertainty relations for the components of angular momentum using the commutators[2] -[6]. For example, we find,

$$
\Delta \mathrm{L}_{\mathrm{x}} \Delta \mathrm{L}_{\mathrm{y}} \geq\left|\frac{\left\langle\left[\mathrm{L}_{\mathrm{x}}, \mathrm{L}_{\mathrm{y}}\right]\right\rangle}{2 \mathrm{i}}\right|=\frac{\hbar}{2}\left\langle\mathrm{~L}_{\mathrm{z}}\right\rangle
$$

\section{Fourier theory:}

The fact that momentum can be expressed as $\mathrm{p}=\mathrm{k} \hbar$ allows us to define a "momentum space" wavefunction that is related to the position space wavefunction via the Fourier transform. A function $\mathrm{f}(\mathrm{x})$ and its Fourier transform. $\mathrm{F}(\mathrm{k})$ are related via the relations:

$$
\begin{aligned}
& f(x)=\frac{1}{\sqrt{2 \pi}} \int_{-\infty}^{\infty} F(k) e^{i k x} d k \\
& F(k)=\frac{1}{\sqrt{2 \pi}} \int_{-\infty}^{\infty} f(x) e^{-i k x} d k
\end{aligned}
$$

These relations can be expressed in terms of $\mathrm{p}$ with a position space wavefunction $\psi(\mathrm{x})$ and momentum space wavefunction $\Phi(\mathrm{p})$ as:

$$
\begin{aligned}
& \psi(\mathrm{x})=\frac{1}{\sqrt{2 \pi \hbar}} \int_{-\infty}^{\infty} \emptyset(\mathrm{p}) \mathrm{e}^{\mathrm{ipx} / \hbar} \mathrm{dp} \\
& \varnothing(\mathrm{p})=\frac{1}{\sqrt{2 \pi \hbar}} \int_{-\infty}^{\infty} \psi(\mathrm{x}) \mathrm{e}^{-\mathrm{ipx} / \hbar} \mathrm{dx}
\end{aligned}
$$

Parseval's theorem tells us that[6]:

$$
\int_{-\infty}^{\infty}|f(x)|^{2} d x=\int_{-\infty}^{\infty}|F(k)|^{2} d k
$$

These relations tell us that $\Phi(\mathrm{p})$, like $\psi(\mathrm{x})$, represents a probability density. The function $\Phi(\mathrm{p})$ gives us information about the probability of finding momentum between $\mathrm{a} \leq \mathrm{p} \leq$ b:

$$
\mathrm{P}(\mathrm{a} \leq \mathrm{p} \leq \mathrm{b})=\int_{\mathrm{a}}^{\mathrm{b}}|\varnothing(\mathrm{p})|^{2} \mathrm{dp}
$$

Parseval's theorem tells us that if the wavefunction $\psi(x)$ is normalized, then the momentum space wavefunction $\Phi(\mathrm{p})$ is also normalized 


$$
\int_{-\infty}^{\infty}|\psi(\mathrm{x})|^{2} \mathrm{dx}=1 \Rightarrow \int_{-\infty}^{\infty}|\varnothing(\mathrm{p})|^{2} \mathrm{dp}=1
$$

It is a fact of Fourier theory and wave mechanics that the spatial extension of the wave described by $\psi(x)$ and the extension of wavelength described by the Fourier transform $\Phi(\mathrm{p})$ cannot be made arbitrarily small. This observation is described mathematically by the Heisenberg uncertainty principle:

$$
\Delta \mathrm{X} \Delta \mathrm{P} \geq \hbar
$$

We can using $\mathrm{p}=\mathrm{k} \hbar, \Delta \mathrm{k} \geq 1$.

Example(2):

A particle of mass $m$ in a one-dimensional box is found to be in the ground state:

$$
\psi(x)=\sqrt{\frac{2}{a}} \sin \left(\frac{\pi x}{a}\right)
$$

Solution:

Using $\mathrm{p}=\mathrm{i} \hbar \mathrm{d} / \mathrm{dx}$ we have:

$$
\mathrm{p} \psi(\mathrm{x})=\mathrm{i} \hbar \frac{\mathrm{d}}{\mathrm{dx}}\left[\sqrt{\frac{2}{\mathrm{a}}} \sin \left(\frac{\pi \mathrm{x}}{\mathrm{a}}\right)\right]=\frac{-\hbar \pi}{\mathrm{a}} \sqrt{\frac{2}{\mathrm{a}}} \cos \left(\frac{\pi \mathrm{x}}{\mathrm{a}}\right)
$$

and:

$$
\begin{gathered}
\mathrm{p}^{2} \psi(\mathrm{x})=\mathrm{i} \hbar \frac{\mathrm{d}}{\mathrm{dx}}\left(\frac{-\hbar \pi}{\mathrm{a}} \sqrt{\frac{2}{\mathrm{a}}} \cos \left(\frac{\pi \mathrm{x}}{\mathrm{a}}\right)\right)=\frac{-\hbar \pi}{\mathrm{a}} \sqrt{\frac{2}{\mathrm{a}}} \sin \left(\frac{\pi \mathrm{x}}{\mathrm{a}}\right) \\
\Rightarrow\langle\mathrm{p}\rangle=\int \psi^{*}(\mathrm{x}) \mathrm{p} \psi(\mathrm{x}) \mathrm{dx}
\end{gathered}
$$

We found in the example above that $\langle p\rangle=0$ for this state.

$$
\begin{gathered}
\left\langle p^{2}\right\rangle=\int \psi^{*}(x) p^{2} \psi(x) d x \\
=\int_{0}^{a} \sqrt{\frac{2}{a}} \sin \left(\frac{\pi x}{a}\right) \frac{\hbar^{2} \pi^{2}}{a^{2}} \sqrt{\frac{2}{a}} \sin \left(\frac{\pi x}{a}\right) d x \\
=\frac{\hbar^{2} \pi^{2}}{a^{2}} \int_{0}^{a}\left(\frac{2}{a}\right)\left[\sin \left(\frac{\pi x}{a}\right)\right]^{2} d x \\
=\frac{2 \hbar^{2} \pi^{2}}{a^{3}} \int_{0}^{a} \frac{1-\cos \left(\frac{2 \pi x}{a}\right)}{2} d x \\
=\left.\frac{\hbar^{2} \pi^{2}}{a^{3}} x\right|_{0} ^{a}=\frac{\hbar^{2} \pi^{2}}{a^{2}} \\
\Delta p=\sqrt{\left\langle p^{2}\right\rangle-\langle p\rangle^{2}}=\sqrt{\frac{\hbar^{2} \pi^{2}}{a^{2}}}=\frac{\hbar \pi}{a} \\
\langle x\rangle=\int \psi^{*}(x) x \psi(x) d x=\int_{0}^{a} \sqrt{\frac{2}{a}} \sin \left(\frac{\pi x}{a}\right) x \sqrt{\frac{2}{a}} \sin \left(\frac{\pi x}{a}\right) d x \\
=\frac{2}{a} \int_{0}^{a} x\left(\sin \left(\frac{\pi x}{a}\right)\right)^{2} d x
\end{gathered}
$$

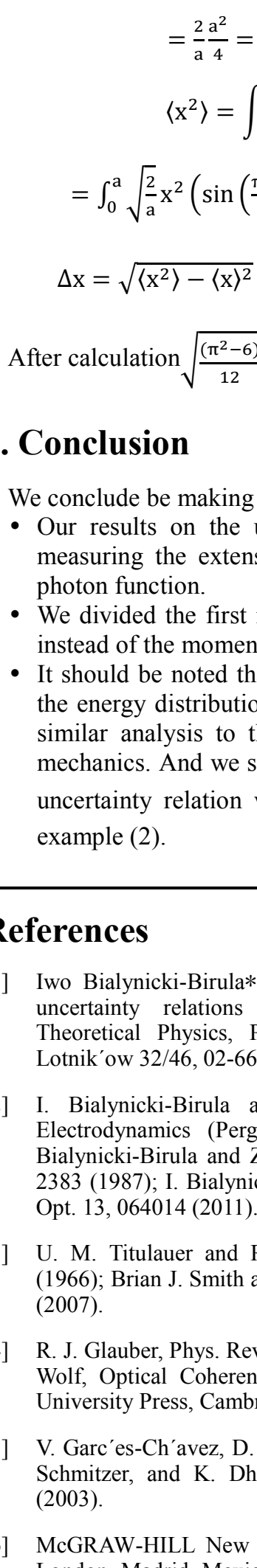

[1] Iwo Bialynicki-Birula*. Zofia Bialynicka-Birula Heisenberg uncertainty relations for photons (2012), Center for Theoretical Physics, Polish Academy of Sciences, Aleja k ow 32/46, 02-668 Warsaw, Poland.

[2] I. Bialynicki-Birula and Z. Bialynicka-Birula, Quantum Electrodynamics (Pergamon, Oxford, 1975), Chap. 9; I. ( Bialynicka-Birula, Phys. Rev. D35, 2383 (1987); I. Bialynicki-Birula and Z. Bialynicka-Birula, J.

[3] U. M. Titulauer and R. J. Glauber, Phys. Rev. 145, 1041 (1966); Brian J. Smith and M. G. Raymer, New J. Phys.9, 414

R. J. Glauber, Phys. Rev. 130, 2529 (1963); L. Man-del and E. Wolf, Optical Coherence and Quantum Optics (Cambridge

V. Garc'es-Ch'avez, D. McGloin, M. J. Padgett, W. Dultz, H.

New York Chicago San Francisco Lisbon London Madrid Mexico City Milan New Delhi San Juan Seoul Singapore Sydney Toronto, 0-07-145546-9. McGrawHill eBooks. Quantum mechanics Demystified.Chap.4,5.

[7] Bialynicki-Birula I and Bialynicka-Birula Z 2006 Beams of electromagnetic radiation carrying angular momentum: the Riemann-Silberstein vector and the classical-quantum correspondence Opt. 
[8] Bialynicki-Birula, photon wave function Progress in Optics, edited by E. Wolf (Elsevier, Amsterdam, 1996), Vol.36; see also ArXiv: quant-ph/0508202.

[9] Bialynicki-Birula I and Bialynicka-Birula Z 1975 Quantum Electrodynamics (Oxford: Pergamon)

[10] "Generalized theory of interference, and its applications," S. Pancharatnam, Proc. Indian Acad. Sci. A 44, 247-262 (1956). See also reference [42] below for the formulation of Pancharatnam's phase in quantum theoretical language.

[11] "The Berry phase as an appropriate correspondence limit of the Aharonov-Anandan phase in a simple model," J. Christian and A. Shimony, in Quantum Coherence, edited by J. Anandan (World Scientific, Singapore, 1990) pp 121-135.

[12] E.P. Wigner in Group Theory and its applications to the Quantum Mechanics of Atomic Spectra, Academic Press, NY(1959); A. Vaglica and G. Vetri, Optics Communications, $51,4(1984) 239$.
[13] J. Schwinger in Quantum theory of Angular Momentum, ed. L. Beidenharn and H. van Dam, Academic Press, NY.(1965)22 K.A. Milton, Rep. Prog. Phys.69.1637 (2006).

[14] K.A. Milton, Rep. Prog. Phys.69.1637 (2006).

[15] McGRAW-HILL New York Chicago San Francisco Lisbon London Madrid Mexico City Milan New Delhi San Juan Seoul Singapore Sydney Toronto, 0-07-145546-9. McGrawHill eBooks. Quantum mechanics Demystified. Chap.6-10.

[16] V.B. Berestetskii, E.M. Lifshitz, and L. P. Pitaevskii, Quantum electrodynamics, 2nd ed. (Pergamon Press Ltd., NY, 1982).

[17] Birulaand Z. Bialynicka-Birula, J.Opt.13, 064014(2011).

[18] U.M. Titulauerand R.J. Glauber, Phys.Rev.145, 1041(1966); Brian J. Smithand M.G. Raymer, New J. Phys.9, 414(2007). 